\title{
Design, Analysis and Control of a Fast Nanopositioning Stage*
}

\author{
Yuen Kuan Yong, Sumeet S. Aphale and S. O. Reza Moheimani \\ School of Electrical Engineering and Computer Science, University of Newcastle, Callaghan, NSW 2308, Australia. \\ Email: \{Yuenkuan.Yong, Sumeet.Aphale, Reza.Moheimani\}@newcastle.edu.au
}

\begin{abstract}
We present a fast flexure-based, piezoelectric stackactuated XY nanopositioning stage which is suitable for highspeed, accurate nanoscale positioning applications. The performance of the design are analyzed using finite-element-analysis software. Experiments demonstrate that the design has a high first resonant mode at $2.7 \mathrm{kHz}$, a low cross-coupling of $\mathbf{- 3 5} \mathbf{~ d B}$ and a relatively large traveling range of $25 \times 25 \mu \mathrm{m}$. These results are in close agreement with the predicted FEA results. Nonlinearities due to hysteresis of the piezoelectric stack actuators are present in the stage. The hysteresis effect is minimized using charge actuation. The Integral Resonant Control (IRC) method is applied to damp the first resonant mode. By implementing feedforward inversion technique, high-speed and accurate scanning performances, up to $400 \mathrm{~Hz}$, are achieved.
\end{abstract}

Index Terms-Nanopositioning stage, finite-element-analysis, Integral Resonant Control, feedforward inversion

\section{INTRODUCTION}

The demand for flexure-based, piezoelectric stack-actuated nanopositioning stages in nano-precision applications, such as nanolithography, scanning probe microscopy, nanometrology, beam steering for optical communication systems, fabrication and assembly of nanostructures [1]-[5] is increasing due to their capability of providing high mechanical bandwidth, large motion range and low cross-coupling between the axes. In the field of cell biology, high bandwidth nanopositioning stages are required to monitor some biological processes that evolve at fast pace [6]. To increase the bandwidth of nanopositioning stages, various approaches have been investigated particularly by improving the mechanical design [7] and by implementing various control algorithms on the systems [8]. In terms of mechanical improvement, the stage is designed to be as compact and rigid as possible in order to achieve high first resonance frequency for each axis [7]. However, the trade-off for a compact design is the reduction in the travel range. An example of this is reported in [9], where the stage has a relatively small travel range of approximately $10 \mu \mathrm{m}$ along the $\mathrm{X}$ and $\mathrm{Y}$ axes, but a high first resonant mode of about $20 \mathrm{kHz}$ for both axes. Multiple piezoelectric stack actuators are arranged in series and in directdrive mode to displace the stage. Most of the commercially available stages have a large travel range (up to $100 \mu \mathrm{m}$ ) but a low first resonance frequency (typically less than $400 \mathrm{~Hz}$ ) and vice versa [10]. In this paper, we report the design of a stage that has a high resonance frequency $(2.7 \mathrm{kHz})$ and a relatively large

\footnotetext{
* This work was supported by the Australian Research Council's Center of Excellence for Complex Dynamic Systems and Control.
}

travel range $(25 \mu \mathrm{m})$. To reduce errors in scanning applications, our stage is designed to have a low cross-coupling of $-35 \mathrm{~dB}$.

The hysteresis in the piezoelectric stack actuators and the lightly damped resonant mechanical modes limit the tracking performance of these nanopositioning stages. Charge actuation has been documented to provide a significant reduction in hysteresis errors [11]. A custom-built charge source capable of driving large capacitive loads such as piezoelectric stack actuators is employed in this work. Accurate tracking is achievable using inversion-based feedforward control [12]. The lightly damped first mechanical resonant mode of the stage makes accurate inversion quite difficult. To improve tracking using the inversion-based feedforward technique, this resonant mode needs to be damped using a suitable feedback controller [13].

Integral Resonant Control (IRC) has been proposed recently to damp collocated systems [14]. It is a simple yet wellperforming technique which adds substantial damping to resonant modes of the system without exciting the high frequency dynamics. In this paper, the IRC technique will be integrated with the feedforward inversion technique to achieve accurate high-speed scanning performances.

This article is organized as follows. In Sec. II, we describe the mechanical design of the nanopositioning stage. This is followed by the FEA results (obtained using ANSYS) of the stage and a discussion on a number of design considerations such as flexure parameters, material used, structural stiffness and piezoelectric stack actuator properties. In Sec. III, the experimental setup and system identification of the stage are presented. Open-loop frequency responses as well as triangular scans are presented to verify that the stage behaves as predicted. Sec. IV presents the design of the IRC as well as the inversionbased feedforward technique. Scanning results in open- and closed-loop are also presented in this section.

\section{Mechanical Design}

The nanopositioning stage presented in this paper has a parallel structure configuration which provides high mechanical stiffness, high mechanical resonance frequency, fast motion speed and high positioning accuracy [15]. The design of the flexure-based nanopositioning stage is based on the concept of compliant mechanisms where motions are generated through the elastic deformation of flexures (flexible elements) [16]. Flexure-based nanopositioning stages provide smooth, repeatable and accurate motions without encountering problems such as wear, backlash, friction and the need for lubrication. Two 
types of flexures are used in the design, circular and beam flexures (see Fig. 1). Piezoelectric stack actuators are commonly used to drive flexure-based stages due to their capability of achieving repeatable nanometer resolution over a very high bandwidth.

The design of the nanopositioning stage consists of two main parts: a) the outer section which consists of amplification levers and circular flexures, and b) the inner section which consists of a stage and beam flexures (see Fig. 1). There are two piezoelectric stack actuators, each providing the required input displacement to the $\mathrm{X}$ and $\mathrm{Y}$ axes respectively. At the outer section, an amplification lever is integrated into each axis of the stage to amplify the displacement of the piezoelectric stack actuator. The amplified motions are transferred to the inner section to displace the platform. Fig. 2 shows the graphical representation of $\mathrm{X}$ and $\mathrm{Y}$ motions of the stage simulated using ANSYS.

Circular flexures are used in the design of the amplification levers since they provide more accurate rotational motions, i.e. the center of deflection can be estimated to be at the center of the circular flexure [17]. A minimum variation of the center of deflection is needed to obtain optimal amplification out of the lever [18]. Circular flexures, which have high off-axis stiffness, are also used to avoid twisting resonant modes (about the $\mathrm{X}$ and $\mathrm{Y}$ axes) that often occur at low frequencies. The amplification ratio of the lever is estimated to be approximately 2.5.

The inner section is designed to minimize the coupling between the $\mathrm{X}$ and $\mathrm{Y}$ motions of the stage. At the inner section, the stage is held by four pairs of beam flexures. The flexures are arranged in such a way so that they are rigid along the direction of motion and are flexible in the axis perpendicular to the motion [7]. Beam flexures are used to guide the stage since they are more flexible and can provide a larger motion range than circular flexures.

\section{A. Design considerations}

Our objective is to design a nanopositioning stage with a high first resonance frequency, a relatively high travel range and a low cross-coupling in motion between the $\mathrm{X}$ and $\mathrm{Y}$ axes. To achieve a high resonance frequency, the design of the stage has to be compact and rigid. As a result, the length of the flexures and the amplification levers have to be small. However, short flexures and levers will reduce the overall $\mathrm{X}$ and $\mathrm{Y}$ motions of the stage. To search for an agreeable compromise between resonance frequency and travel range, ANSYS is used to conduct a number of design iterations until the desired design criteria is achieved. The thicknesses of all the flexures are carefully taken into consideration to minimize the crosscoupling, and to maximize both the resonance frequency and travel range of the stage.

The resonance frequency of the stage is also dependent on the type of the material used. A material with high Young's modulus of elasticity $E$ to density ratio $\rho$ is preferred because a stiff and light material (high $E$ and low $\rho$ ) will improve the mechanical stiffness and bandwidth of the stage. Aluminium

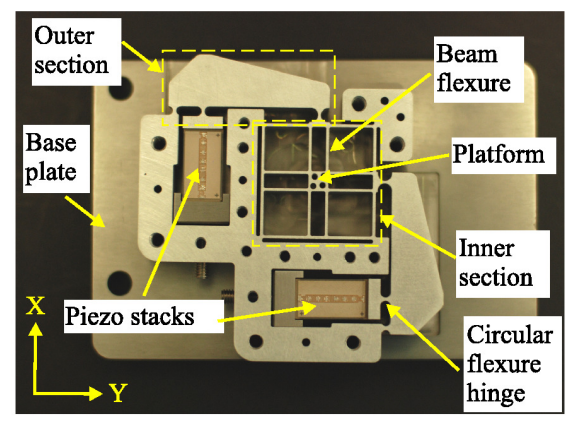

Figure 1. Flexure-based XY nanopositioning stage. The inner section is designed to minimize the coupling between the $\mathrm{X}$ and $\mathrm{Y}$ motions. The outer section is designed to amplify the displacement of piezoelectric stack actuators.

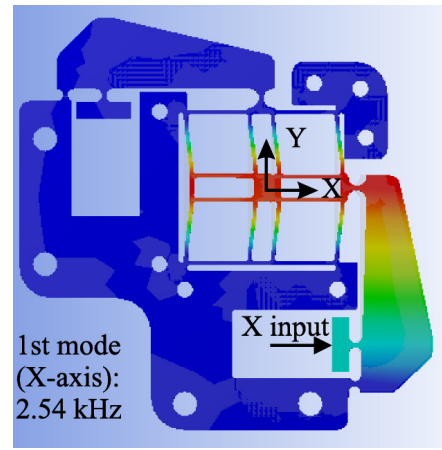

Figure 2. ANSYS simulations of the nanopositioning stage along the X-axis. The simulations of the Y-axis are not shown as there are similar.

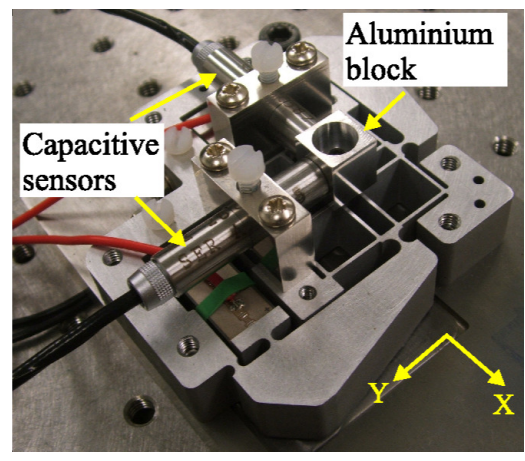

Figure 3. Experimental setup of the XY nanopositioning stage. Capacitive sensors are incorporated to measure the $\mathrm{X}$ and $\mathrm{Y}$ motions of the stage. The aluminium block is machined to have a fine surface finish and is used as the sensing target.

alloy 7075 (Al 7075), with $E=72 \mathrm{GPa}$ and $\rho=2.81 \mathrm{~g} / \mathrm{cm}^{3}$ (a relatively high value of $E / \rho$ ), is used to fabricate the stage.

The structural stiffness $\left(k_{s}\right)$ of the stage (which is experienced by the piezoelectric stack actuator) is also carefully considered in the design process. This is because the maximum displacement of the piezoelectric stack actuator is governed by $k_{s}$, i.e. $\Delta L=\Delta L_{o}\left(k_{\text {piezo }} /\left(k_{s}+k_{\text {piezo }}\right)\right)$ [10], where $\Delta L_{o}$ is the maximum nominal displacement of a piezoelectric stack actuator without external spring load, $\Delta L$ is the displacement with external spring load, $k_{s}$ is the structural stiffness of the 


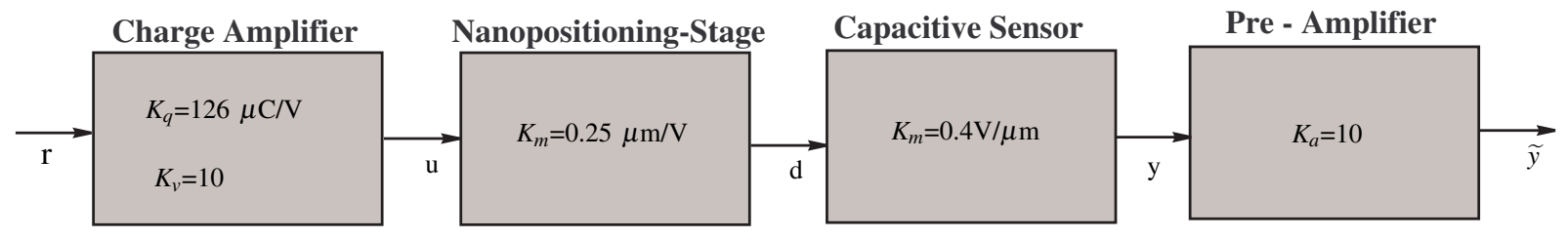

Figure 4. Gains associated with the experimental setup. $r$ is the reference input in volts. $u$ is the output of the charge amplifier in $\mu \mathrm{C}$ used as the driving input for the nanopositioning stage. $d$ is the actual displacement of the nanopositioning stage in $\mu \mathrm{m}$ and $y$ is the proportionally scaled capacitive sensor output in volts. A gain of 10 was introduced during measurement using a pre-amplifier to improve the resolution and minimize quantization noise of the dSPACE ADC/DAC channels.

stage, $k_{\text {piezo }}$ is the piezoelectric stack actuator stiffness. In order to increase the resonance frequencies of the stage, a high value of $k_{s}$ is required. However, the increase of the stiffness will result in a decrease in the maximum displacement $(\Delta L)$ of the piezoelectric stack actuator. To reach an acceptable compromise between the resonance frequency and the travel range of the stage, $k_{s}$ is chosen to be approximately $20 \%$ of $k_{\text {piezo }}$. For the best result, a piezoelectric stack actuator with large $k_{\text {piezo }}$ is selected; thus the stage can be designed to have a large $k_{s}$ and high mechanical resonance frequency without losing too much of its travel range. A piezoelectric stack actuator (P-888.50 from Physik Instrumente) is selected for the design. The $k_{\text {piezo }}$ of this piezoelectric stack actuator is $200 \mathrm{~N} / \mu \mathrm{m}$ which is well-suited for the design requirement aforementioned.

The final design of the stage is predicted to have a first resonance frequency of $2.5 \mathrm{kHz}$, a travel range of $25 \mu \mathrm{m}$ and a cross-coupling of $-35 \mathrm{~dB}$. The FEA simulation of the first resonance frequency along the $\mathrm{X}$-axis of the stage can be found in Fig. 2. For brevity, FEA simulations of the Y-axis are not presented as there are similar to the $\mathrm{X}$-axis.

\section{EXPERIMENTAL SETUP AND SySTEM IDENTIFICATION}

In this section, we describe the experimental setup and characterization of the XY nanopositioning stage. Using the design results predicted by ANSYS, the stage is fabricated using Al 7075 with a thickness of $12.8 \mathrm{~mm}$. WEDM (wireelectrical-discharge-machining) technique is used to fabricate the stage due to its accuracy and precision [19]. The stage is mounted on a $10 \mathrm{~mm}$ steel plate to be rigidly held in place. A small aluminium block with a fine surface finish is attached to the nanopositioning stage as shown in Fig. 3. This block is used as the target for displacement sensing. It also serves as a stage over which a sample can be placed and moved. Two ADE Technologies 8810 capacitive sensors (which have a static gain of $2.5 \mu \mathrm{m} / \mathrm{V}$ ) are placed in close proximity to the adjacent surfaces of the aluminium block to measure the displacements along the $\mathrm{X}$ and $\mathrm{Y}$ axes.

Fig. 3 shows the experimental setup used for this work. To clarify the associated gains of the system, a block diagram is presented in Fig. 4. A Stanford Research Systems Pre-amplifier was used to improve the resolution of the dSPACE ADC/DAC boards and minimize quantization noise. The pre-amplifier gain was set to 10 but was taken out of the data when plotting, thus the D.C. gain of the system from Digital Signal Analyzer (DSA) input to capacitive sensor output is unity $(0 \mathrm{~dB})$ as shown in Fig. 5 . The piezoelectric stack actuators were driven by the charge amplifier that has a gain of $126 \mu \mathrm{C} / \mathrm{V}$ and an equivalent voltage gain of 10. Hysteresis due to the use of piezoelectric stack actuators is minimized using charge actuation. The benefit of using charge actuation is demonstrated in Fig. 6. Without using the charge actuation, the hysteresis loop is approximately $3 \mu \mathrm{m}$ wide ( $12.6 \%$ of the maximum stage displacement) compared to $0.33 \mu \mathrm{m}(1.6 \%)$ when the charge actuation is used.

To identify the linear model within the bandwidth of interest, the plant was identified using a bandlimited random noise input of amplitude $300 \mathrm{mVpk}$ within the frequency range of 10 $\mathrm{Hz}$ to $10 \mathrm{kHz}$, using a $\mathrm{HP} 35670 \mathrm{~A}$ dual channel spectrum analyzer. The stage is a two-input two-output system. Input signals applied to the piezoelectric stacks in the $\mathrm{X}$ and $\mathrm{Y}$ directions are denoted as $u_{x}$ and $u_{y}$ (in volts) respectively, and the outputs are the respective stage displacements $d_{x}$ and $d_{y}$ (in $\mu \mathrm{m}$ ) given by the capacitive sensors as corresponding voltages. Random noise input signals $\left(u_{x}\right.$ and $\left.u_{y}\right)$ generated by the spectrum analyzer are applied to the $\mathrm{X}$ - and Y-axis piezoelectric stack actuators respectively through the charge amplifier. The capacitive sensor outputs $\left(d_{x}\right.$ and $\left.d_{y}\right)$ are measured and fed back respectively to the spectrum analyzer to construct the corresponding frequency responses. The transfer functions of the frequency response can be described as $G_{x x}(j \omega)=d_{x}(j \omega) / u_{x}(j \omega), G_{y x}=d_{y}(j \omega) / u_{x}(j \omega), G_{x y}(j \omega)=$ $d_{x}(j \omega) / u_{y}(j \omega)$ and $G_{y y}(j \omega)=d_{y}(j \omega) / u_{y}(j \omega)$. Here, $u_{x}(j \omega)$, $u_{y}(j \omega), d_{x}(j \omega)$ and $d_{y}(j \omega)$ denote the Fourier transforms of $u_{x}$, $u_{y}, d_{x}$ and $d_{y}$ respectively. Fig. 5 plots the frequency responses of the stage.

In Fig. 5, the first resonant peak of the stage (at both axes) is observed at $2.7 \mathrm{kHz}$, about $8 \%$ higher than the predicted value of ANSYS simulations. The magnitude of the crosscoupling terms $G_{y x}$ and $G_{x y}$ are about $-35 \mathrm{~dB}$ and $-40 \mathrm{~dB}$ less than that of $G_{x x}$ and $G_{y y}$ respectively. The measured $G_{y x}$ is in close agreement with that obtained from ANSYS simulations. However there are some differences between the $G_{x y}$ values. These are most likely due to the fact that piezoelectric stack actuators are not properly modeled. The differences could also be partially due to manufacturing tolerances and machining imperfections [20].

The scanning range of the stage is measured by applying 


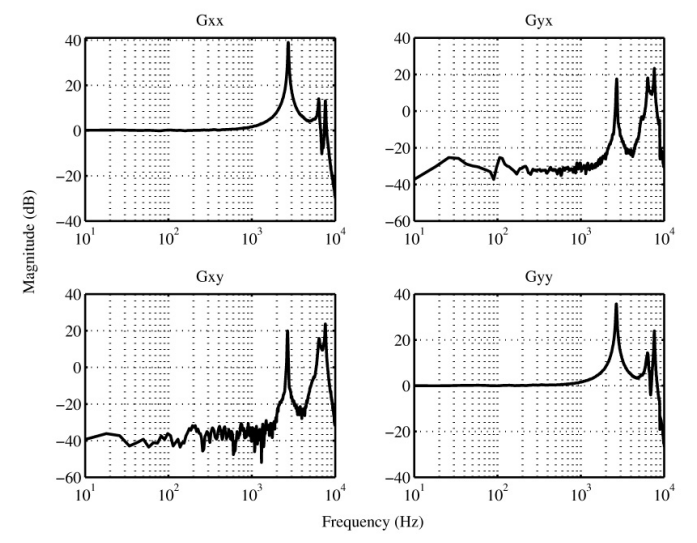

Figure 5. Measured frequency responses of the nanopositioning stage. The input is reference voltage $r$ and the output is capacitive sensor voltage $y$ corresponding to stage displacement $d$ in $\mu \mathrm{m}$. Note that the nanopositioning stage is driven using a charge amplifier with a gain of $126 \mu \mathrm{C} / \mathrm{V}$.
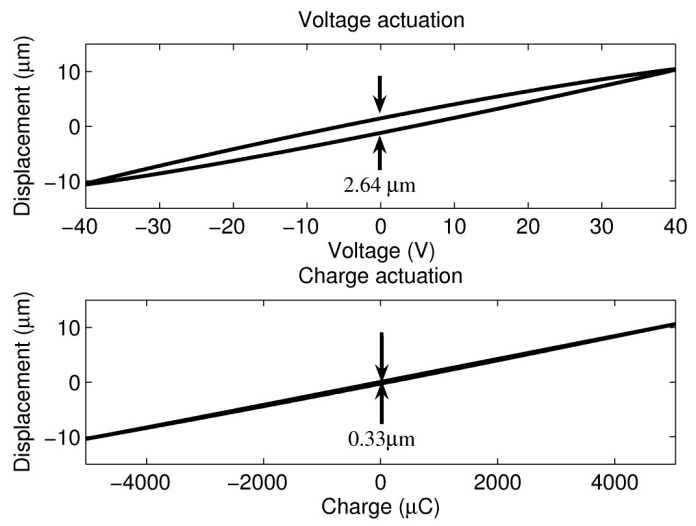

Figure 6. Hysteresis loops for voltage $(\mathrm{V})$ and charge $(\mu \mathrm{C})$ actuations.

a low frequency triangular signal with a peak to peak voltage equal to the maximum voltage that could be applied to this specific piezoelectric stack actuator, i.e. $100 \mathrm{~V}$. As the piezoelectric stack was driven using charge, it was ascertained that the maximum voltage across the piezoelectric stack did not go beyond $100 \mathrm{~V}$. The measured travel range is $25 \mu \mathrm{m}$ which is in close agreement to the predicted value from ANSYS analysis.

Open-loop traces of a triangular waveform are obtained at 10, 20, 30 and $40 \mathrm{~Hz}$ (see Fig. 7). Earlier stage designs produced acceptable scans at very low frequencies $(f \leq 1 \mathrm{~Hz})$ [10]. It can be seen clearly that the stage produces open-loop scans accurately at frequencies of 10 and $20 \mathrm{~Hz}$. The $30 \mathrm{~Hz}$ triangular input excites the first resonance of the stage. This effect is more prominent in the $40 \mathrm{~Hz}$ scan. To increase the positioning bandwidth of the stage, both damping and tracking control strategies need to be implemented. In the next section, the details of the IRC scheme for damping and the inversion-based feedforward scheme for tracking are presented.

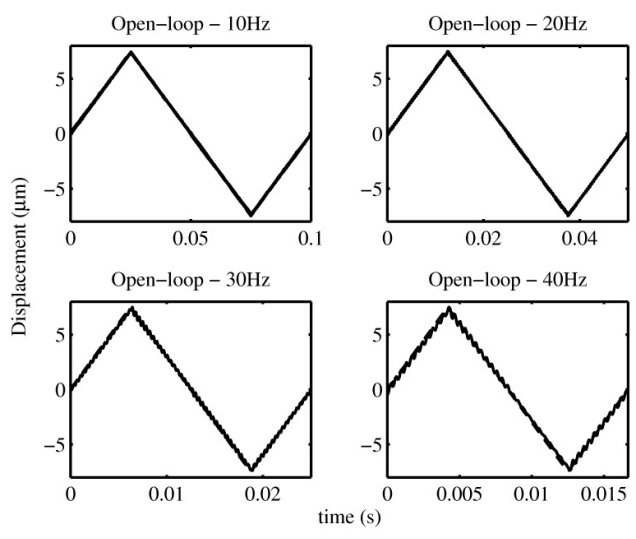

Figure 7. Open-loop tracking performance at 10, 20, 30 and $40 \mathrm{~Hz}$. Triangular reference signal (dashed line) and output signal (solid line) are plotted. The first resonant mode of the stage is excited at $30 \mathrm{~Hz}$.

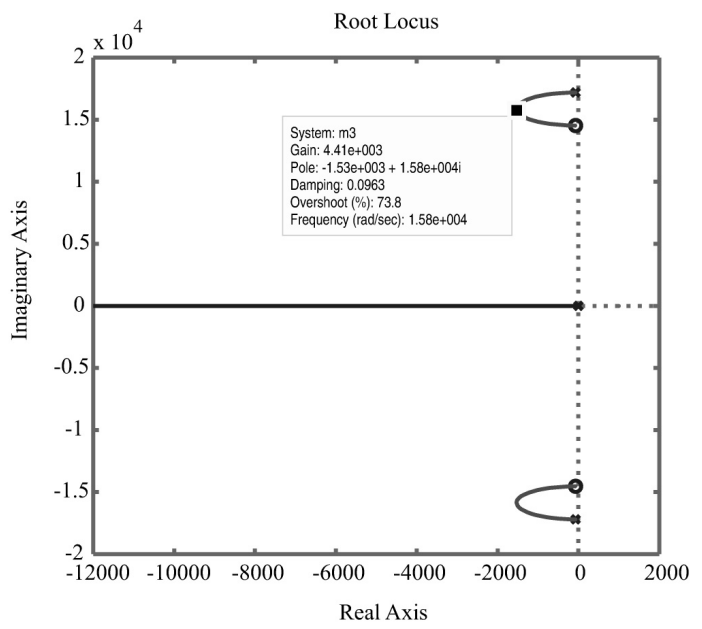

Figure 8. The root-locus plot of the loop shows the trajectory where the pole travels towards the zero when the gain increases. Note that maximum damping is achieved when the system gain is 4410 . This gain is implemented in the actual setup.

\section{Control Design}

The dominant resonant peak of the stage occurs at 2.73 $\mathrm{kHz}$ and has a dynamic range of $39 \mathrm{~dB}$. As the other two modes of the identified system are quite far away from the first one and have a much lower dynamic range, only the first mode is prioritized. The IRC scheme is an effective method of introducing substantial damping to the system. It also has a desirable property of not exciting the higher-frequency dynamics. The method followed to implement a suitable IRC scheme is discussed below.

Step 1: A second order model is fitted to the frequency response of the plant such that it accurately captures the dominant resonant mode of the stage. The transfer function of this model is given by,

$$
G_{\text {model }}=\frac{-0.01 s^{2}-1.376 s+2.929 \times 10^{8}}{s^{2}+137.6 s+2.958 \times 10^{8}}
$$




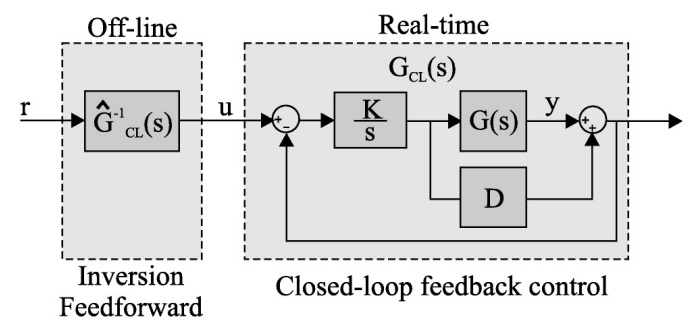

(a)

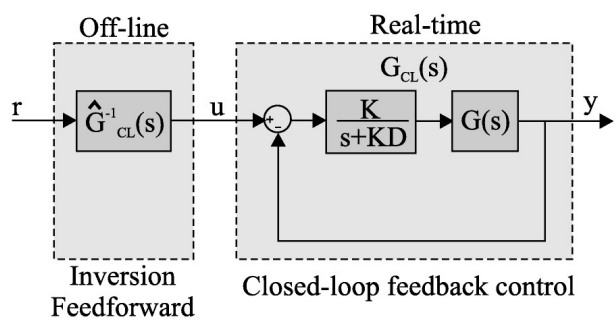

(b)

Figure 9. Block diagrams of the IRC scheme with inversion feedforward. (a) The IRC with inversion feedforward. (b) The simplified IRC which has a low-pass filter characteristic. Note that both $\mathrm{K}$ and $\mathrm{D}$ are negative in this case.

\begin{tabular}{ccc}
\hline System & Poles & Zeros \\
\hline$G_{\text {model }}$ & $-68.8 \pm 1.72 \times 10^{4} i$ & $\pm 3.85 \times 10^{4}$ \\
$G_{\text {model }}+D$ & $-68.8 \pm 1.72 \times 10^{4} i$ & $-68.8 \pm 1.45 \times 10^{4} i$ \\
\hline
\end{tabular}

Table I

LOCATION OF POLES AND ZEROS FOR THE SECOND-ORDER PLANT MODEL $G_{m o d e l}$ AND THE OVERALL SYSTEM WiTH THE FEEDTHROUGH TERM $G_{\text {model }}+D$.

Step 2: Table I gives the location of the poles and zeros for the second-order plant model $G_{\text {model }}$ and the overall system with a feedthrough term $G_{\text {model }}+D$. Using a suitable feedthrough term, $D$, the right half-plane zero can be shifted to the left half-plane, thus making the overall plant $G_{\text {model }}+D$ conducive to integral feedback. In this case, a feedthrough term of $D=-4$ when added to the system will render it minimum phase.

Step 3: A unity gain negative integrator $\left(\frac{-1}{s}\right)$, was simulated in negative feedback with the overall plant and a root-locus plot was obtained, see Fig. 8. A gain of 4410 achieves maximum damping.

Thus, for Fig. 9(a) and (b), $K=-4410$ and $D=-4$. The overall controller has a low-pass filter type characteristics and the system can be simplified to the one shown in Fig. 9(b). Fig. 10 , shows the simulation results obtained using the measured open-loop frequency response data.

The implemented control scheme damps the dominant resonant mode effectively by $28 \mathrm{~dB}$, see Fig. 10 . These results match with the simulation results shown in Fig. 10 quite accurately. The plant has been identified up to $10 \mathrm{kHz}$ and the control scheme does not have any adverse effect on the high frequency dynamics. Note that the DC gain of the closedloop system is 0.4 , i.e. to get one unit output, the closedloop system needs an input of 2.5 units. Also note that as $\mathrm{K}=-4410$ (negative), the output is $180^{\circ}$ out of phase with the input. The gain as well as the phase shift are automatically taken into account by the feedforward inversion technique. The control strategy was implemented using a dSPACE-1005 rapid prototyping system equipped with 16-bit ADC (DS2001) / DAC (DS2102) cards. To ensure that there are no aliasing effects, a sampling frequency of $40 \mathrm{kHz}$ was chosen.
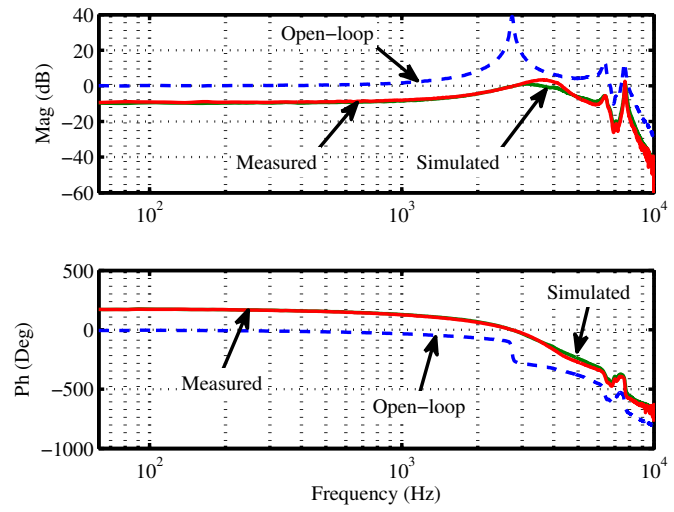

Figure 10. Simulated and measured frequency responses of the closed-loop damped system with the IRC scheme. The simulated results match with the closed-loop results.

\section{SCANNing Results}

In this section, the scanning performance of the stage is evaluated using triangular waveforms. The inversion-based feedforward approach is based on accurate model identification. After examining the closed-loop data, it was deemed feasible to invert the model in the frequency range of $0 \mathrm{~Hz}$ to 5.4 $\mathrm{kHz}$. To evaluate the high-speed scanning performance of the nanopositioning stage, triangular waveforms with fundamental frequencies of 100, 200, 300 and $400 \mathrm{~Hz}$ were chosen. The open-loop scans are plotted in Fig. 11. Inversion-based inputs are obtained by using all the odd harmonics of the fundamental frequency of the triangular wave that lie within the bandwidth of $0 \mathrm{~Hz}$ to $5.4 \mathrm{kHz}$. Thus, the $100 \mathrm{~Hz}$ inversion-based input is obtained using the first 54 harmonics and the $200 \mathrm{~Hz}$ input is obtained using the first 27 harmonics. Similarly, 18 harmonics are included in the $300 \mathrm{~Hz}$ input and 13 harmonics are included in the $400 \mathrm{~Hz}$ input. Fig. 12 plots the closed-loop scan obtained. It is clear that the tracking performance of the stage in closedloop is substantially superior to that in open-loop.

Open- and closed-loop scans are plotted for charge actuation. To ascertain that the current limit of the charge amplifier is not exceeded, the scans were limited to $15 \mu \mathrm{m}$. Table II documents the RMS scanning error in nanometers, in open- and closedloop for $90 \%$ of the scanning range $(13.5 \mu \mathrm{m})$. The tracking 


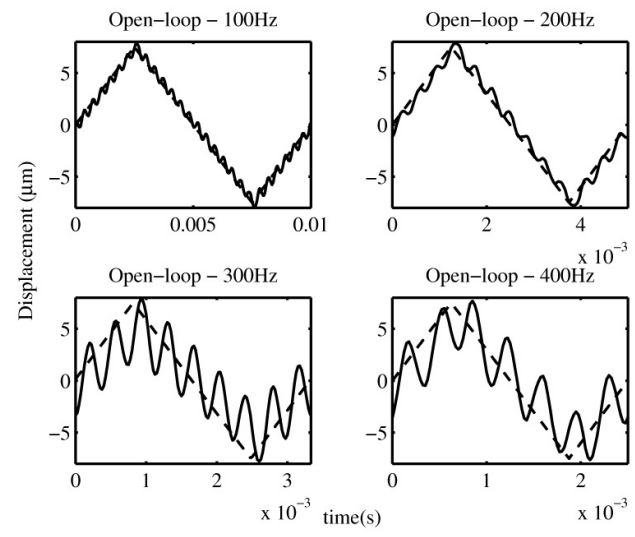

Figure 11. Open-loop tracking performance at 100, 200, 300 and $400 \mathrm{~Hz}$. Triangular reference signal (dashed line) and output signal (solid line) are plotted.

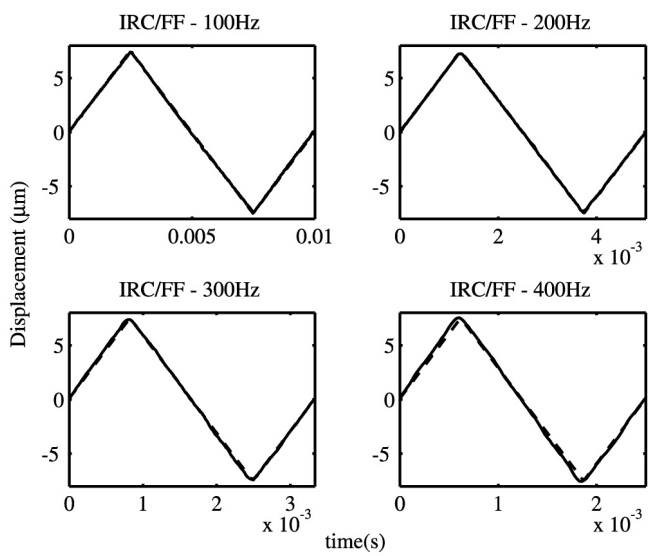

Figure 12. Closed-loop tracking performance at 100, 200, 300 and $400 \mathrm{~Hz}$. Triangular reference signal (dashed line) and output signal (solid line) are plotted.

as a percentange of the scanning range is also presented in the table.

\section{CONCLUSIONS}

The nanopositioning stage is designed to fulfil three main requirements, which are to achieve a) high bandwidth, b) relatively high motion range, and c) low cross-coupling. ANSYS is used to analyze the design and the experimental results

\begin{tabular}{ccccc}
\hline RMS error $(\mathrm{nm})$ & $100 \mathrm{~Hz}$ & $200 \mathrm{~Hz}$ & $300 \mathrm{~Hz}$ & $400 \mathrm{~Hz}$ \\
\hline Open-loop & 447 & 679 & 2254 & 2432 \\
IRC+Feedforward & 51 & 60 & 150 & 259 \\
\hline \% error & $100 \mathrm{~Hz}$ & $200 \mathrm{~Hz}$ & $300 \mathrm{~Hz}$ & $400 \mathrm{~Hz}$ \\
\hline Open-loop & 2.98 & 4.65 & 15.03 & 16.21 \\
IRC+Feedforward & 0.38 & 0.44 & 1.11 & 1.92 \\
\hline
\end{tabular}

Table II

RMS AND PERCENTAGE ERRORS OF THE TRACKING PERFORMANCE IN OPEN- AND CLOSED-LOOP FOR $90 \%$ OF THE SCANNING RANGE. are in close agreement with the predicted FEA values. By implementing the IRC and feedforward technique, accurate high-speed scans up to $400 \mathrm{~Hz}$ were achieved. The mechanical design of the stage and its associated controller design will be refined to achieve faster scan rates and reduced coupling between various axes. The ultimate goal is to reach scan rates suitable for generating real time video with the high precision.

\section{REFERENCES}

[1] S. Salapaka, A. Sebastian, J. P. Cleveland, and M. V. Salapaka, "High bandwidth nano-positioner: A robust control approach," Rev. Sci. Instrum., vol. 73, no. 9, pp. 3232-3241, 2002.

[2] K.-B. Choi and J. J. Lee, "Passive compliant wafer stage for single-step nano-imprint lithography," Rev. Sci. Instrum., vol. 76, p. 075106, 2005.

[3] S. Gonda, T. Kurosawa, and Y. Tanimura, "Mechanical performances of a symmetrical, monolithic three-dimensional fine-motion stage for nanometrology," Meas. Sci. Technol., vol. 10, pp. 986-993, 1999.

[4] J. J. Gorman, N. G. Dagalakis, and B. G. Boone, "Multi-loop control of nanopositioning mechanism for ultra-precision beam steering," in Proc. SPIE Conf. Free-Space Laser Communication and Active Laser Illumination III, San Diego, CA, vol. 5160, 2003, pp. 170-181.

[5] S. Verma, W. jong Kim, and H. Shakir, "Multi-axis maglev nanopositioner for precision manufacturing and manipulation applications," IEEE Trans. Industry Applications, vol. 41, no. 5, pp. 1159-1167, 2005.

[6] T. Ando, N. Kodera, D. Maruyama, E. Takai, K. Saito, and A. Toda, "A high-speed atomic force microscope for studying biological macromolecules in action," Japanese Journal of Applied Physics, vol. 41, no. 7B, pp. 4851-4856, 2002.

[7] J. H. Kindt, G. E. Fantner, J. A. Cutroni, and P. K. Hansma, "Rigid design of fast scanning probe microscopes using finite element analysis," Ultramicroscopy, vol. 100, pp. 259-265, 2004.

[8] A. J. Fleming and S. O. R. Moheimani, "Sensorless vibration suppression and scan compensation for piezoelectric tube nanopositioners," IEEE Trans. Contr. Syst. Tech., vol. 14, no. 1, pp. 33-44, 2006.

[9] G. Schitter, K. J. Åstrom, B. DeMartini, P. J. Thurner, K. L. Turner, and P. K. Hansma, "Design and modeling of a high-speed AFM-scanner," IEEE Trans. Contr. Syst. Tech., vol. 15, no. 5, pp. 906-915, 2007.

[10] MicroPositioning, NanoPositioning, NanoAutomation. Solutions for Cutting-Edge Technologies. Physik Instrumente Catalog, 2001.

[11] A. J. Fleming and S. O. R. Moheimani, "A ground-loaded charge amplifier for reducing hysteresis in piezoelectric tube scanners," Rev. Sci. Instrum., vol. 76, no. 7, p. 073707, 2005.

[12] Q. Zhou and S. Devasia, "Preview-based optimal inversion for output tracking: Application to scanning tunneling microscopy," IEEE Trans. Contr. Syst. Tech., vol. 12, no. 3, pp. 375 - 386, 2004.

[13] S. S. Aphale, S. Devasia, and S. O. R. Moheimani, "High-bandwidth control of a piezoelectric nanopositioning stage in the present of plant uncertainties," Nanotechnology, vol. 19, p. 125503 (9pp), 2008.

[14] S. S. Aphale, A. J. Fleming, and S. O. R. Moheimani, "Integral resonant control of collocated smart structures," Smart Materials and Structures, vol. 16, pp. 439-446, 2007.

[15] Y. K. Yong, T.-F. Lu, and D. C. Handley, "Review of circular flexure hinge design equations and derivation of empirical formulations," Precision Engineering, vol. 32, no. 2, pp. 63-70, 2008.

[16] L. Howell, Compliant Mechanisms. John Wiley \& Sons, Inc., 2001.

[17] N. Lobontiu, J. S. N. Paine, E. Garcia, and M. Goldfarb, "Corner-filleted flexure hinges," Transactions of the ASME, Journal of Mechanical Design, vol. 123, pp. 346-352, 2001.

[18] M. Jouaneh and R. Yang, "Modeling of flexure-hinge type lever mechanisms," Precision Engineering, vol. 27, pp. 407-418, 2003.

[19] K. H. Ho, S. T. Newman, R. S., and R. D. Allen, "State of the art in wire electrical discharge machining (WEDM)," International Journal of Machine Tools and Manufacture, vol. 44, no. 12-13, pp. 1247-1259, 2004.

[20] J. W. Ryu and D.-G. Gweon, "Error analysis of a flexure hinge mechanism induced by machining imperfection," Precision Engineering, vol. 21, pp. 83-89, 1997. 